\title{
Altered Proinflammatory Cytokines Distinguish Elderly with Major Depressive Disorder from Amnestic Mild Cognitive Impairment in a Chinese Population
}

Jing Nie

Shanghai Mental Health Center https://orcid.org/0000-0002-9102-2488

\section{Yuan Fang}

Shanghai Mental Health Center

\section{Qi Qiu}

Shanghai Mental Health Center

\section{Yuan Li}

Shanghai Mental Health Center

\section{Lu Zhao}

Shanghai Mental Health Center

Xiang Liu

Shanghai Mental Health Center

Xia Li (D ja_1023@hotmail.com )

Shanghai Mental Health Center https://orcid.org/0000-0003-3531-0861

\section{Research Article}

Keywords: late life depression, amnestic mild cognitive impairment, Neuro-inflammation, cytokines

Posted Date: June 8th, 2021

DOI: https://doi.org/10.21203/rs.3.rs-583849/v1

License: (9) (i) This work is licensed under a Creative Commons Attribution 4.0 International License. Read Full License 


\section{Abstract}

Background: Research evidence has shown that late life depression(LLD)and amnestic mild cognitive impairment(aMCl)are both related to dementia and usually accompanied by inflammatory dysregulation, but few studies have compared the levels of chemokines and cytokines between these two disorders. In this cross-sectional study, we aimed at identifying differences in neuro-inflammation between patients with prodromal stage of $A D$ (amnestic mild cognitive impairment, aMCl; Late life depression LLD) and the normal controls (NC).Additionally, the potential association between cognitive function and cytokines characteristics was evaluated.

Methods: Using Luminex assays,we simultaneously measured 29 cytokines,including 6 CXC chemokine ligand(CXCL5,CXCL6,CXCL8,CXCL9,CXCL11,CXCL16), 15 CC chemokine ligand(CCL1, CCL2,CCL3,CCL7, CCL8, CCL11, CCL13,CCL15,CCL17, CCL19,CCL20, CCL23, CCL24, CCL25, CCL27), IL-13, MIP-2, IFN-ץ, VCAM-1, IL-2, IL-4, TNF-a and IL-6 in plasma of subjects. Demographics, cognitive function and lifestyle factors were also collected. All subjects( $n=69,23$ each group)were age- and gender-matched outpatients with LLD or aMCl without dementia(based on DSM-IV criteria)and normal controls(NC)in community. The Chinese versions of Montreal Cognitive Assessment(MoCA) and neuropsychological test battery (NTB) were used to evaluate the cognitive function in participants.

Results: LLD patients showed remarkably higher level of IL-6 than the aMCI and $\mathrm{NC}$ groups. We also observed five CC chemokines (CCL2,CCL17,CCL15, CCL13and CCL5) and four CXCs (CXCL5,CXCL6,CXCL16 and CXCL11) were decreased in patients with LLD. In addition, CXCL5 and VCAM1 were correlated with age and clinical characteristic with gender difference. Importantly, using two parameters(IL-6 and CCL13) could effectively distinguish LLD from aMCl with an area under curve of $0.809(95 \% \mathrm{Cl} 0.68-0.93)$.

Conclusion: Under conditions of neuro-inflammation, distinct dysregulated pro-inflammatory cytokine and chemokines were associated with patients with predementia stage LLD and aMCI.. Our study showed that the model based on the pro-inflammatory cytokine could distinguish LLD from aMCl. These novel findings will give new clues to provide new therapeutic target for intervention or a marker for two diseases with similar pre-dementia syndromes.

\section{Introduction}

This is expected that by 2050, the annual cost of dementia in the United States alone may exceed US $\$ 600$ billion. Alzheimer's disease (AD) is the most common types of dementia which was recognized by the World Health Organization as a global public health priority ${ }^{[1]}$. There were no effective medical treatments to cure the disease, however the prevalence of dementia would be halved if its onset were delayed by 5 years ${ }^{[2]}$. Therefore, the prodromal stage of $A D$ was a great opportunity for effective treatment and postponing the disease onset. 
Late-life major depressive disorder(LLD)showed significant cognitive impairment, decreased activity, primary motivational impairment and show less mood symptoms ${ }^{[3,4]}$. Currently many Meta-analyses showed the strong association between late life depression and an increased risk for Alzheimer's disease $(A D)$ and it might be an etiological factor for $A D^{[5]}$. The concept of mild cognitive impairment is used to describe confirmed subjective and neuropsychological cognitive deficits of persons whose ability of daily living is not altered. Comparing with $3 \%$ of the population without mild cognitive impairment at the same age, around $39 \%$ mild cognitive impairment patients develop dementia over the subsequent 3 to 10 years $^{[6]}$. Mild cognitive impairment can be divided into non-amnestic mild cognitive impairment $\square \mathrm{MCl}[$ and amnestic mild cognitive impairment subtypes $\square \mathrm{aMCl}$. The aMCl subgroup showed typical impairment of episodic memory and represents the most common prodromal stage of $A D^{[7]}$.

Pro-inflammatory cytokines and chemokines have attracted increased attention as potential mediators of interactions between the immune and neuroendocrine systems and specific pathways involved in mood, cognition, and energy. Based on pervious researches, patients receiving cytokines treatment have a greater risk of developing depressive disorder, and administration of anti-cytokines to patients with inflammatory disease and depression could relieve depressive symptoms ${ }^{[8,9]}$. Inflammation may also play an important role in psychiatric disorders in elderly because a pro-inflammatory state is associated with pathological of aging. The classification of the Inflammation and clinical characteristics in late life depression and amnestic mild cognitive impairment patients may shed light on the pathogenesis of their conversion to dementia.

Cytokines play important roles in neuro-inflammation. Previous researches had investigated that proinflammatory cytokines such as interleukin-6 (IL-6), interleukin-1 (IL-1), and tumor necrosis factor-alpha (TNF-a) were significantly different between LLD and normal elderly or dementia and $\mathrm{MCl}{ }^{[10,11]}$. Chemokines including $\mathrm{C}, \mathrm{C}-\mathrm{C}, \mathrm{C}-\mathrm{X}-\mathrm{C}$ and $\mathrm{CX} 3 \mathrm{C}$ chemokines are a family of small cytokines. They play key roles to induce chemotaxis, promote differentiation and multiplication of leukocytes, and cause tissue extravasation ${ }^{[12]}$. Recent data has suggested its clinical significance in central nervous system (CNS), like the multiple sclerosis (MS), neuro-borreliosis (NB) and Alzheimer's disease ${ }^{[13,14]}$. However very few studies in the literature have concerned about the levels of chemokines in LLD and aMCI. Josje failed to find a significant difference in the levels of chemokine ligand CCL2, and CCL4 in studies with individuals: 88 patients with aMCl and 154 patients with $A D^{[15]}$. With regard to $L L D$, previous studies showed that depression was significantly associated with higher levels of IL-1 $\beta$ and CXCL8 in plasma and cerebrospinal fluid ${ }^{[16,17]}$. Josef et al compared the levels of CXCL8,CCL7,CCL15,CCL18,CCL5 among 16 patients with Alzheimer's disease, 16 patients with mild cognitive impairment patients, 35 patients with depression and 19 healthy controls. Patients with $\mathrm{MCl}$ had higher levels of CCL5,CCL18 versus depression and healthy control. No statistically significant difference was found between LLD and control groups. The research didn't distinguish the $\mathrm{MCl}$ subtype and analyse enough chemokines ${ }^{[18]}$. To our knowledge there are no studies directly examining the difference of pro-inflammatory cytokines and chemokines among patients with $\mathrm{aMCl}$ and LLD, and healthy controls. This gap in the literature led us to 
investigate the difference of general immune dysfunction in two high probably prodromal stage of $A D$ (ie.LLD, aMCl) and healthy controls.

In this study, we aimed to characterize the neuro-inflammation in two subtypes of patients with preclinical dementia stage (late life depression, LLD; amnestic mild cognitive impairment, aMCI), compared with the sex and age-matched normal controls (NC). We hypothesized that patients with LLD and aMCI would show different characteristics in inflammatory dysfunction and were associated with clinical parameters.

\section{Materials And Methods}

\subsection{Subjects and Clinical Classification}

Twenty-three participants diagnosed with amnestic mild cognitive impairment and twenty-three healthy control were concurrently recruited from four different communities in Shanghai. The same amount of geriatric depression patients were subjected to the diagnosis according to the DSM-IV diagnostic standard by clinicians from out-patients. People with other psychiatric illness, neurological, a history of major diseases (e.g. autoimmune disease, myocardial infarction, stroke, cancer, HIV, and hepatitis B or C) or with recent illness or infection were excluded.

\subsection{Diagnostic Procedures}

All participants received clinical diagnoses established by three different geriatric psychiatrists according to the results of standardized assessment and review. Clinical diagnosis of aMCl were adapted from Petersen et $a^{[19]}$, requiring evidence of definite decline in memory ( memory complaintscorroborated by an informant, MoCA scores of $>1.5 \mathrm{SD}$ of age-appropriate norms or abnormal memory function for age) and additional without significant impact on daily living, severity of symptoms or consequent functional limitation not meeting the DSM-IV dementia standard; a diagnosis of normal control was made if participants demonstrated no evidence of cognitive decline as compared to their baseline cognitive functions on clinical interview and assessment. Patients with late life depression were diagnosed according to Diagnostic and Statistical Manual of Mental Disorders, Fourth Edition, Text Revision (DSMIV-TR) criteriawith depression onset occurs over 60 years old .Participants were excluded when they had a dementia diagnosis or were suspected to have dementia based on clinicians' judgment. All the participants were matched according to the mean age and sex. Then, a detailed medical, social, and family history was obtained from each participant. All participants completed the following subtests: CMoCA (Chinese Version of Montreal Cognitive Assessment) , C-NTB (Chinese Version of neuropsychological test battery) including WMDS (Wechsler Memory digit span), CFT (Category Fluency Test), COWAT (Controlled Word Association Test) and Geriatric Depression Scale (GDS). This study was approved by the Institution Review Board of the Shanghai Mental Health Center. Written informed consent was obtained from all the participants or their representatives.

\subsection{Sample collection and preparation}


All the participants fasted for $10 \mathrm{~h}$ prior to venous peripheral blood sampling.Fasting blood samples were collectedto determine CRP. After centrifugation $\left(2500 \mathrm{rpm}, 15 \mathrm{~min}\right.$, at $\left.4^{\circ} \mathrm{C}\right)$, plasma was collected from EDTA tubes, then stored at $-80^{\circ} \mathrm{C}$ until the assays were performed.

\section{Luminex Assays}

Using a Luminex microbeads, we simultaneously measure the concentration of 29 cytokines and chemokines(Table 1). The plasma samples were thawed at $4^{\circ} \mathrm{C}$ and were centrifuged at $1500 \mathrm{rpm}$ to remove any aggregate protein which could potentially obstruct the measurement. The human Magnetic Luminex Performance Assay (R\&D Systems, no. LXSAHM-33, USA) were performed according to the manufacturer's protocol. A X200 (Luminex, Austin, TX) was used to read the multiplex assay.

Table 1

The results of 29 human chemokines

\begin{tabular}{|lllll|}
\hline CCL1/TCA-3 & CCL2/ MCP-1 & CCL3/ MIP-1 a & CCL7/ MCP-3 & CCL8 /MCP-2 \\
\hline CL11/Eotaxin & CCL13/MCP-4 & CCL15/MIP-18 & CCL17/TARC & CCL19/MIP-3 $\beta$ \\
\hline CCL20/MIP-3 a & CCL23/MPIF-1 & CCL24/Eotaxin-2 & CCL25/ TECK & CCL27/ CTACK \\
\hline CXCL5/ENA-78 & CXCL6/GCP-2 & CXCL8/IL-8 & CXCL9/MIG & CXCL11/I-TAC \\
\hline CXCL16/SCYB16 & IL-1 $\beta$ & MIP2 & IFN-Y & VCAM-1 \\
\hline TNF-a & IL-2 & IL-4 & IL-6 & \\
\hline
\end{tabular}

\subsection{Statistical Analysis}

Statistical analysis was performed using SPSS Version 22 and GraphPad Prism software (version 6.0; GraphPad, Inc.). Statistically significant differences among groups were assessed by one-way ANOVA ,and the pairwise differences between groups were determined by a post hoc test.If the assumption of normality was violated, non-parametric test was applied. We also performed partial correlation analysis to assess the associations between chemokines and pro-inflammatory cytokines of men and women as independent variables and MoCA scores as dependent variables. Receiver operating characteristic (ROC) analysis was used to evaluate the ability of cytokinesto discriminatepatients with LLD from aMCl. Statistical significance was set at $p<0.05$ on a two-tailed test.

\section{Results}

\subsection{Demographic and clinical characteristics}

Twenty-three subjects each group(healthy control subjects, aMCI, LLD) took part in our study. There was no significantly difference between any of the groups in age and male-to-female ratio.Years of educations were higher in the healthy control group compared with other two groups. In lifestyle, lower proportion of patients with late life depression had habits in taking regular exercise or drinking tea.As expected, the 
control group had a significantly higher mean c-MoCA scores than the all other groups, the WMDS , CFT, COWAT scores were also lower in the $\mathrm{aMCl}$ and depression groups compared with healthy control. The Geriatric Depression Scale identified that the depression group had a significantly higher scores than the control and $\mathrm{aMCl}$ groups(Table 2).

Table2 Comparison of demographic characteristics and cognition function between patients with late life depression (LLD), patients with amnestic mild cognitive impairment $(\mathrm{aMCl})$, and healthy controls (Controls) 


\begin{tabular}{|c|c|c|c|c|}
\hline & $\begin{array}{l}\text { aMCl } \\
(n=23)\end{array}$ & $\begin{array}{l}\text { LLD } \\
(n=23)\end{array}$ & $\begin{array}{l}\text { Controls } \\
(n=23)\end{array}$ & Statistics \\
\hline \multicolumn{5}{|l|}{ Clinical demographic data } \\
\hline \multirow[t]{2}{*}{ Mean (sd) age } & $73.0(4.5)$ & $71.9(5.7)$ & $72.5(0.8)$ & $F=0.41$ \\
\hline & & & & $P=0.665$ \\
\hline \multirow[t]{2}{*}{ Means (sd) years of education } & $13.0(3.4)$ & 11.2(2.9)\# & 15.2(2.2)\#\& & $F=11.11$ \\
\hline & & & & $P<0.01$ \\
\hline \multirow[t]{2}{*}{ Gender (male: female) } & $10: 13$ & $8: 15$ & 9:14 & $x 2=0.37$ \\
\hline & & & & $P=0.833$ \\
\hline \multicolumn{5}{|l|}{ Lifestyle (Y:N) } \\
\hline \multirow[t]{2}{*}{ Smoking } & $5: 18$ & $3: 20$ & $3: 20$ & $\chi 2=0.87$ \\
\hline & & & & $P=0.649$ \\
\hline \multirow[t]{2}{*}{ Drinking } & $6: 17$ & $4: 19$ & 4:19 & $x 2=4.66$ \\
\hline & & & & $P=0.097$ \\
\hline \multirow[t]{2}{*}{ Tea drinking habits } & $13: 10$ & $5: 18^{\star} \#$ & $12: 11$ & $\chi 2=7.50$ \\
\hline & & & & $P=0.024$ \\
\hline \multirow[t]{2}{*}{ Regular exercise } & $21: 2$ & $6: 17 * \#$ & $17: 6$ & $\chi 2=22.71$ \\
\hline & & & & $P<0.01$ \\
\hline \multicolumn{5}{|l|}{ Global Cognitive scales } \\
\hline \multirow[t]{2}{*}{ Means (sd) C-MoCA score } & $20.8(2.6)$ & $18.8(6.1)$ & 26.3(2.1)\#\& & $F=21.10$ \\
\hline & & & & $P<0.01$ \\
\hline \multirow[t]{2}{*}{ Means (sd) WMDS score } & $12.1(2.8)$ & $13.4(3.1)$ & 16.6(3.3) \#\& & $F=10.38$ \\
\hline & & & & $P<0.01$ \\
\hline \multirow[t]{2}{*}{ Means (sd) CFT score } & $6.7(2.5)$ & $5.5(3.6)$ & 10.0(4.2) \#\& & $F=10.11$ \\
\hline & & & & $P<0.01$ \\
\hline \multirow[t]{2}{*}{ Means (sd) COWAT score } & $12.1(3.4)$ & 9.3(3.5) \# & 14.8(3.3) \#\& & $F=15.26$ \\
\hline & & & & $P<0.01$ \\
\hline \multirow[t]{2}{*}{ Geriatric Depression Scale } & $7.0(3,9)$ & $22.0(4.6) * \#$ & $5.1(2,7)$ & $F=80.82$ \\
\hline & & & & $P<0.01$ \\
\hline
\end{tabular}


Notes: SD, standard deviation. All values, except Gender, Anti-inflammatory medication and lifestyle are expressed as mean SD.

*Significantly different from controls $(P \leq 0.05)$.

\#Significantly different from aMCl group $(\mathrm{P} \leq 0.05)$.

\&Significantly different from LLD group ( $\mathrm{P} \leq 0.05)$.

aMCl, amnestic mild cognitive impairment; LLD, late life depression; Control, normal elderly; c-MoCA, Chinese Version of Montrea/Cognitive Assessment; WMDS , Wechsler Memory digit span; CFT, Category Fluency Test; COWAT, Controlled Word Association Test;

\subsection{Cognitive assessment}

The comparison of sub item of c-MoCA score among the three groups was shown in Fig1.

All function domains with the exception of the orientation and visual spatial function were significantly changed between late life depression and control group. The depression group similarly showed poorer performance in executive function and attention compared with amnestic mild cognitive impairment group. However, late life depression patients performed better than a $\mathrm{MCl}$ subjects in short-term memory test.[VS function $((\mathrm{H}(2)=4.207, \mathrm{p}=0.122)$, Executive function $(\mathrm{H}(2)=35.707, \mathrm{p}<0.001)$, ACW $(\mathrm{H}(2)=$ 17.141, $p<0.001)$, Language $(H(2)=13.421, p=0.001)$, orientation to time and place $(H(2)=1.775$, $\mathrm{p}=0.412)$, short-term memory $(H(2)=26.225, \mathrm{p}<0.001))($ Fig. 2$)$.

\subsection{Inflammatory protein levels}

As revealed in Fig2(A-J) shows the protein plasma concentrations for the pro-inflammatory cytokines and chemokines. Totally, patients in late life depression groups had significantly higher level of IL-6(2.17 \pm 0.12 vs. $2.86 \pm 0.21$ vs. $2.28 \pm 0.23, F=3.81, P<0.05$ Fig. $2(A))$ than the control or aMCl subjects.LLD patients show lower levels of CXCL5 (339.39 \pm 46.53 vs. $605.36 \pm 100.40, F=3.32, P<0.05$ Fig. $2(B)$ ), CXCL6(121.62 \pm 13.08 vs. $220.99 \pm 39.90, F=3.52, \mathrm{P}<0.05$ Fig. $2(\mathrm{C})), \mathrm{CXCL} 16(112.60 \pm 9.89$ vs. 170.14 $\pm 25.45, \mathrm{~F}=2.98, \mathrm{P}<0.05$ Fig. $2(\mathrm{D}))$ and CCL25(145.17 \pm 12.91 vs. $205.17 \pm 27.24, \mathrm{~F}=2.30, \mathrm{P}<0.05 \mathrm{Fig}$. $2(E))$ than control group. Furthermore, aMCl group showed higher level of CCL13(2.17 \pm 0.12 vs. 2.86 \pm 0.21 vs. $2.28 \pm 0.23, F=3.81, P<0.01$ Fig. $2(F))$ than the control or depression subjects.

Comparing with depression patients, aMCl group had higher levels ofCCL17(170.97 \pm 25.13 vs. $115.55 \pm$ $9.74, F=2.10, P<0.05$ Fig. $2(\mathrm{G})), C C L 15(6048.00 \pm 752.43$ vs. $4908.22 \pm 791.82, F=2.91, P<0.05$ Fig. 2(H)), CCL2(216.32 \pm 24.57 vs. $153.19 \pm 15.11, F=2.39, P<0.05$ Fig. $2(I)), C X C L 11(33.90 \pm 1.73$ vs. $28.31 \pm 1.96, F=2.51, P<0.05$ Fig. $2(J))$ in plasma. Meanwhile, no significant differences were noticed in plasma concentrations for other chemokines and cytokines among three groups (Table3). 
Table3 Level of inflammatory protein concentration in controls and patients 


\begin{tabular}{|c|c|c|c|c|}
\hline & $\mathrm{aMCl}(\mathrm{n}=23)$ & $\operatorname{LLD}(n=23)$ & Controls $(n=23)$ & Statistics \\
\hline \multirow[t]{2}{*}{ TNF-a邓pg/ml } & $5.2(1.8)$ & $5.4(1.4)$ & $5.5(0.9)$ & $F=0.24$ \\
\hline & & & & $P=0.7886$ \\
\hline \multirow[t]{2}{*}{ CCL1囚pg/ml囚 } & $1.7(0.9)$ & $1.6(0.8)$ & $1.4(0.5)$ & $F=0.67$ \\
\hline & & & & $P=0.515$ \\
\hline \multirow[t]{2}{*}{ CXCL8囚pg/mI } & $3.2(2.2)$ & $6.7(2.9)$ & $3.5(2.8)$ & $F=1.61$ \\
\hline & & & & $P=0.207$ \\
\hline \multirow[t]{2}{*}{ CCL7囚pg/mI } & 20.3(16.9) & 13.6(13.1) & 17.7(15.8) & $F=1.17$ \\
\hline & & & & $P=0.316$ \\
\hline \multirow[t]{2}{*}{ MIP2囚pg/ml囚 } & $194.8(140.4)$ & $180.6(260.8)$ & 268.9(259.4) & $F=1.04$ \\
\hline & & & & $P=0.361$ \\
\hline \multirow[t]{2}{*}{ 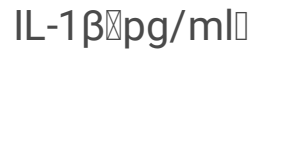 } & $3.7(1.7)$ & $4.5(2.7)$ & $3.3(2.5)$ & $F=1.49$ \\
\hline & & & & $P=0.234$ \\
\hline \multirow[t]{2}{*}{ IFN-ү冈pg/ml] } & 26.6(10.4) & $24.9(6.0)$ & $24.2(6.4)$ & $F=0.54$ \\
\hline & & & & $P=0.588$ \\
\hline \multirow[t]{2}{*}{ CCL20『pg/ml『 } & $60.4(29.5)$ & $55.6(38.2)$ & $64.3(54.6)$ & $F=0.30$ \\
\hline & & & & $P=0.741$ \\
\hline \multirow[t]{2}{*}{ CCL24『pg/mI } & $550.5(224.8)$ & $446.4(260.2)$ & $553.1(292.4)$ & $F=1.04$ \\
\hline & & & & $P=0.361$ \\
\hline \multirow[t]{2}{*}{ 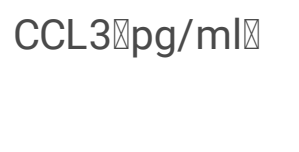 } & $133.8(32.5)$ & 127.4(41.5) & $130.3(49.4)$ & $F=0.10$ \\
\hline & & & & $P=0.902$ \\
\hline \multirow[t]{2}{*}{ CCL8囚pg/mI } & 279.5(16.7) & 278.4(13.1) & 275.7(14.9) & $F=0.44$ \\
\hline & & & & $P=0.645$ \\
\hline \multirow[t]{2}{*}{ IL-4『pg/ml区 } & $29.2(6.5)$ & $28.3(5.0)$ & $27.5(5.9)$ & $F=0.43$ \\
\hline & & & & $P=0.652$ \\
\hline \multirow[t]{2}{*}{ CCL19『pg/ml } & 115.9(38.8) & 158.4(143.9) & 114.4(64.6) & $F=0.70$ \\
\hline & & & & $P=0.505$ \\
\hline \multirow[t]{2}{*}{ IL-2】pg/mI区 } & $4.0(1.9)$ & $3.3(2.5)$ & $3.6(2.2)$ & $F=0.43$ \\
\hline & & & & $P=0.656$ \\
\hline
\end{tabular}




\begin{tabular}{|c|c|c|c|c|}
\hline CXCL9邓pg/ml囚 & $588.1(80.7)$ & 611.6(81.4) & 613.2(89.3) & $\begin{array}{l}F=0.14 \\
P=0.869\end{array}$ \\
\hline \multirow[t]{2}{*}{ CCL23》pg/ml囚 } & $421.5(150.6)$ & $454.6(216.4)$ & $458.5(200.2)$ & $F=1.71$ \\
\hline & & & & $P=0.198$ \\
\hline \multirow[t]{2}{*}{ VCAM-1『ng/ml } & $1210.8(452.7)$ & $1342.2(594.8)$ & $1138.4(599.4)$ & $F=1.66$ \\
\hline & & & & $P=0.208$ \\
\hline \multirow[t]{2}{*}{ CCL27》pg/ml囚 } & $508.8(146.1)$ & 488.2(138.0) & $508.5(143.2)$ & $F=0.87$ \\
\hline & & & & $P=0.431$ \\
\hline \multirow[t]{2}{*}{ CCL11囚pg/ml囚 } & $85.4(31.5)$ & 73.7(17.4) & $76.5(42.2)$ & $F=0.22$ \\
\hline & & & & $P=0.806$ \\
\hline \multirow[t]{2}{*}{$\mathrm{CRP}, \mathrm{mg} / \mathrm{mL}$} & $1.58(0.3)$ & $1.92(0.47)$ & $2.38(0.50)$ & $F=1.51$ \\
\hline & & & & $P=0.230$ \\
\hline \multicolumn{5}{|c|}{ 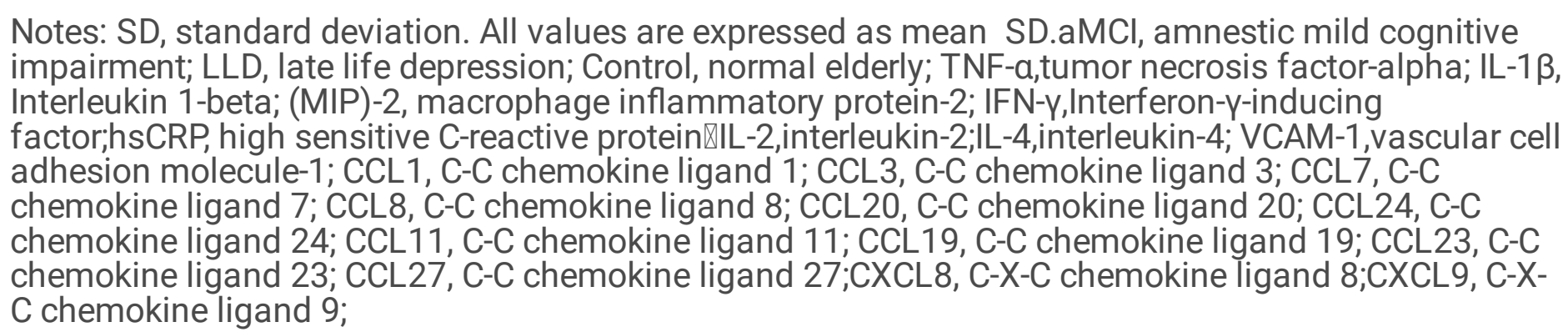 } \\
\hline
\end{tabular}

\subsection{Relationships of potential neuro-inflammation markers between depression mood and cognitive function as well as depression and aging}

Partial correlations between variables of interest for whole sample, controlling for age and years of education in Fig3. As shown in the Venn diagram, in red, represents dysfunction chemokines andproinflammatory cytokineswhich might be risk markers for the cognitive impairment. The yellow one represents dysfunction chemokines and cytokines, which showpossible relationship with depression response. The chemokines, which might be markers for aging, were showing in the green circle. In male participants, cognition and depression shared CXCL5, respectively, corresponding to points in which the areas overlap. One chemokine, VCAM-1 is in B and C in female one. Thec-MoCAand GDS scores were used to determine the cognitive impairment, depression severity in subjects.

\subsection{Discriminant Validity: ROC}

The results of the ROC analyses, which assessed the ability of Chemokines and pro-inflammatory cytokines concentrations discriminate between late life depression and $\mathrm{aMCl}$ groups are presented in 
Figure4.

For IL-6 plasma level, to distinguish aMCI participants from LLDpatients, the area under the curve (AUC) was found to be $0.748(95 \% \mathrm{Cl} 0.60-0.89)$. An optimal statistical cutoff is achieved at a cut-off score of $2.59 \mathrm{pg} / \mathrm{ml}$ (56.5\% sensitivity, 91.3\% specificity)showing in Figure4(A).

For CCL13 plasma level, to distinguish aMCI participants from LLDpatients, the area under the curve (AUC) was found to be 0.711 (95\% Cl 0.55-0.87). An optimal statistical cutoff is achieved at a cut-off score of $52.67 \mathrm{pg} / \mathrm{ml}$ (60.9\% sensitivity, $87.0 \%$ specificity)showing in Figure4(B).

Combining two parameters above(using logistic regression analysisP $=\mathrm{e}^{\mathrm{x}} /\left(1+\mathrm{e}^{\mathrm{x}}\right), \mathrm{X}=-0.765+(1.196 \times \mid \mathrm{L}-6)+$ (0.044×CCL13), the area under the curve (AUC) was found to be 0.809 (95\% Cl 0.68-0.93). An optimal statistical cutoff is achieved at a cut-off score of 0.50 (78.3\% sensitivity, $69.6 \%$ specificity)showing in Figure4(C).

\section{Dissusion}

To our knowledge, our study, with multiple types of cytokines, is one of the first time to characterize neuro-inflammation of Chinese elder patients in the different clinical stages of dementia, classified as LLD, and aMCI. We found that the dysfunction cytokines in patients of aMCI and LLD was markedly different, and the altered chemokines was significantly correlated with the clinical parameters, which were potential markers for cognitive impairment, depression severity, and aging with different gender. Importantly, the discriminating models based on two combined parameters(IL-6 and CCL13) could effectively distinguish LLD from aMCI patients. The novel results of this study will give new leads to further understand the aetiology of pre-clinical stage of dementia and provide therapeutic target for intervention or a marker for this disease.

Multiplex analytical technologies are quite crucial to the complex task of deciphering disease-specific biomarker patterns as they provide opportunities for an all-inclusive approach, which was not possible using traditional techniques such as enzyme-linked immunosorbent assay (ELISA). In our study, using a luminex assay, a reliable multiplex analytical technologies, it could measure multiple different cytokines simultaneously in a single run of the assay with small sample-size requirements ${ }^{[20]}$. Simultaneous measurement of multiple cytokines in the peripheral blood sample provides a cost- and time-effective strategy to resolve complex interactions among signaling molecules to obtain an objective pattern of numerous cytokines within one experiment, which provides a more inclusive and comprehensive depiction of pre-clinical stage of dementia ${ }^{[21]}$ showing in Table1.

Cytokines are typically produced by cells of the immune system upon activation, which play key roles in the development and control of immune responses. Chemokines represent one of the largest subfamilies of cytokines, which have been divided into several sub-groups bases on their chemical structures. CXC and $\mathrm{CC}$ were two major subfamilies, depending on whether the first two $\mathrm{N}$ - terminal cysteines have an amino acid between them $(C X C)$ or are adjacent $(C C)^{[22]}$. Although chemokines have been relatively 
neglected in the investigations of mechanisms of psychiatric disorders, recent evidence has begun to demonstrate an association between chemokines and neurobiological processes which have been strongly implicated in the pathogenesis and pathophysiology of psychiatric disorders. Dysfunction cytokines have been associated with both recent $A D$ and other psychiatric disorders, including mild cognitive impairment, schizophrenia, depression and bipolar disorder ${ }^{[23]}$. Although the exact effect of the pro-inflammatory cytokines in $A D$ remains unknown, more and more evidence suggests that inflammation is involved in pathological process of $A D$, and plays a crucial role in the early stages of disease when intervention maybe most beneficial ${ }^{[24]}$.

Our study benefited from LLD, aMCl and healthy control groups that were well matched for age and gender. All groups demonstrated no use of anti-inflammatory drug. Changes of cytokine levels were compared among three groups. A group of chemokines including one pro-inflammatory cytokines(IL6), five CC chemokines (CCL2, CCL13, CCL15, CCL17, and CCL25) and

four CXCs (CXCL5,CXCL6,CXCL11 and CXCL16) were dysregulated in patients with prodromal stage of AD. We demonstrated significantly lower levels of CXCL5,CXCL6,CXCL16 and CCL25 in LLD compared with control group. CCL2,CCL13, CCL15, CCL17, and CXCL11 were significantly higher in case-aMCI than LLD, while IL-6 was significantly higher in case-LLD than in case-aMCl and control(Fig. 2).

The first dysregulated cytokine IL-6 has been extensively investigated in many preclinical and clinical studies as a pro-inflammatory cytokine which can accelerate ongoing neurodegenerative processes in AD. Pervious research found that plasma circulating IL- 6 may be a useful indication for future cognitive function ${ }^{[25]}$, and increased Interleukin-6 (IL-6) level was reported in late life depression patients in peripheral blood ${ }^{[26]}$.Leung $\mathrm{R}$ show elevated IL-6 in AD patients versus controls and negatively correlated with MMSE scores ${ }^{[27]}$ however we did not find significant associations between IL- 6 concentration and cognition. Then, we briefly discuss the information about following cytokines in general. CCL2 and CCL13 belong to the monocyte chemotactic protein (MCP) family, which exert potent pro-inflammatory actions through chemotaxis of monocyte-derived macrophages and other inflammatory leukocytes to the inflamed or injured central nervous system (CNS). CCL2 has also been proved to have effects on microglia, inducing migration and proliferation. CCL5, a target gene of NF-KB activity, is expressed by T lymphocytes, macrophages, platelets, synovial fibroblasts, tubular epithelium, and certain types of tumor cells. CCL15 is classified as a macrophage inflammatory protein and has a pro-inflammatory effect. CCL17 have been shown to be downstream mediators of the effects of systemic interferons on inflammatory state at the choroid plexus, and are correlated with hippocampal neurogenesis and hippocampal-dependent learning and memory tasks in aging. CXCL5 is a pro-inflammatory cytokine involved in both innate and acquired immune response. CXCL6 is an important inflammatory cytokine that recruits inflammatory cells to the site of inflammation by binding to the receptors CXCR1 and CXCR2. CXCL11 is a ligand of CXCR3, which have clearer detrimental pro-inflammatory effects by mediating chemotaxis of natural killer cells, Th1 cells, and their associated classically activated (M1) proinflammatory monocyte-derived macrophages. CXCL16 is expressed as not only a membrane-bound 
molecule but also a soluble chemokine, which has been identified to bind with the chemokine receptor CXCR6 to regulate immune cell chemotaxis into CXCL16-enriched environments ${ }^{[28-30]}$.

Elevated cytokines have also been found in the CSF and plasma of patients with AD, particularly in mild cognitive impairment patients ${ }^{[31]}$. The reduction of some chemokines in LLD patients which promote the activation of macrophages, natural killer cells, Th1 cells in our study is not consistent with increased inflammation of the nervous system in $\mathrm{MCl}$. It suggested that patients in pre-clinical stage of dementia with similar cognitive deficits showed significantly different levels of pro-inflammatory cytokines. In line with our finding, suicide people reported a similar decrease in the levels chemokines (eg.CCL1, CCL8, CCL13, CCL15, CCL17, CCL19, CCL20, CXCL11) ${ }^{[32]}$. Indeed suicidal ideation decreases with aging, but if older people have suicidal thoughts they are at a higher risk of actually committing suicide than any other age and these rates continue to rise in many countries ${ }^{[33]}$. The data indicate that these cytokines might predict who would most likely attempt or commit suicide after exposure to a depression situation.

The demographic characteristics in the LLD group, which probably would affect inflammatory feature. Personal characteristics and a series of complex factors (Low economic status, cultural, poor physical health and social isolation) would affect whether a person, group, or community lead to a late-onset major depressive disorder. In this study, the results showed that $26.1 \%$ of the elderly led a sedentary lifestyle, while $91.3 \%$ mild cognitive impairment subjects performed exercise habits. Depressive symptoms are associated with decreased physical performance in older adults in both cross-sectional and longitudinal epidemiological studies ${ }^{[34,35]}$. However, the relationship between increasing proportion of having physical activity and decreasing symptoms in depression may be bidirectional. On the one hand, depression may lead to decreased levels of activity due to low motivation and energy. On the other hand, the decreasing exercise could be a risk factor for depression. Exercises are known to increase the secretion of multiple hormones which had antidepressant function: physical activities can change norepinephrine activity in central system temporarily, decrease the hypo-thalamopituitary-adrenocortical axis, and increase the secretion of beta-endorphins ${ }^{[36,37]}$. To some extent, the lower levels of chemokine may also be explained by the finding that late life depression patients with a sedentary and lacking of stimulation lifestyle.

Past studies have demonstrated that sex has a significant impact on the functions of the immune system. The production of cytokines and chemokines by innate immune cells also differs between the sexes $^{[38,39]}$. In our study, potential cytokine markers for aging, depression and cognition were identified with different gender. Interestingly, there are two CXC dysregulated chemokines associated with aging process and cognition function, one $\mathrm{CC}$ chemokine related to depression in male. In female, we reported that seven dysregulated cytokines correlated with the parameters reflecting the cognitive function (MoCA scores), two CC chemokines and VCAM-1 concerned with depression, and three dysregulated cytokines relevant to aging. We also found the overlap of expression of potential chemokines among the tested group(Fig. 3). Aging is the strongest risk factor for neurodegenerative diseases, it results in a significant change in inflammatory mediators ${ }^{[40]}$. In our study, CXCL5 was up-regulated in aging and inversely 
associated with MoCA scores in male. The alteration is particularly interesting because it played an important role in maintenance of synaptic formation in the hippocampus of male rats ${ }^{[41]}$. It is known that hippocampus is one of the most critical regions in brain, highly important to cognitive function and affective behavior. Endothelial cell VCAM-1, is a member of the immunoglobulin superfamily, upregulated on endothelium in response to inflammation. Many studies have demonstrated that plasma VCAM-1 levels increase significantly with age, and have been reported in community-dwelling elderly people with symptoms of depression. Most studies reported female gender as one of the main predictors of depression in late life, our results suggesting the differences between genders may contribute to separate dysregulated cytokine which may lead to cognitive function or depression in pre-clinical stage of dementia ${ }^{[42,43]}$.It may encourage further investigation of the mechanisms of dysregulated chemokines in cognitive impairment and depression studies considering different gender in the context of aging.

Here, we demonstrated that the using IL-6, CCL13 and together could clearly distinguish pre- dementia stage LLD from aMCl, with AUCs of $0.75,0,71$ and 0.81 . Treating AD can be challenging, because the pathological changes have begun in many years before the diagnosis of clinical dementia. Due to the lack of drug slow the progression of dementia, therapies should be developed to target on people at risk of dementia. More importantly, researches before investigated the efficacy of antidepressants for the treatment of depression in patients with both depression and dementia. Only two of seven studies found antidepressant pharmacotherapy to be more effective than placebo. Meanwhile, little is known about the treatment of depression in patients with $\mathrm{MCl}$, rigorous identification of $\mathrm{MCl}$ in geriatric major depression treatment trials will help to advance effectiveness of treatments ${ }^{[44,45]}$.Thus, the distinguished cytokine will facilitate detection of people with cognitive impairment, allowing for further investigation of the proinflammatory cytokine effects on the pre-clinical $A D$ and neurodegeneration diseases, adopt the individuation preventive and therapeutic measure.

The following limitations of our study have to be discussed. It is undeniable that the cross-sectional study design and small sample size limits causal conclusions regarding the relationship between inflammation and late life depression. Moreover, several methods for predicting the progression of $\mathrm{MCl}$, such as positron emission tomography (PET) and analysis of biomarkers in the cerebrospinal fluid. However, these methods are unacceptable in the elderly for their high costs and invasive nature. Here, the individuals were recruited from our cohort who has been diagnosed by three different geriatric psychiatrists according to Petersen criteria in follow up for three years. The criteria was wide used and has been reported with reasonable accuracy.

Moreover, it is undeniable that further longitudinal studies combined with proteomic and genetic markers are required.

\section{Conclusions}

In conclusion, this is the first report estimating plasma cytokine profile in LLD in comparison with in aMCl patients and controls. Dysregulated chemokines may represent powerful candidate biomarkers, which 
can predicate aging, cognitive function, and depression. Our study showed that the model based on the pro-inflammatory cytokine could distinguish LLD from aMCl.

These results may suggest involvement of different immunological mechanisms in two diseases with similar pre-dementia syndromes, showing that cytokines may benefit developing approaches for preclinical dementia diagnosis and further treatment.

\section{Abbreviations}

\begin{tabular}{|ll|}
\hline aMCl & Amnestic mild cognitive impairment \\
\hline LLD & Late life depression \\
\hline NC & Normal controls \\
\hline C-MoCA & Chinese Version of Montrea/ Cognitive Assessment \\
\hline GDS & Geriatric depression Scale. \\
\hline WMDS & Wechsler Memory digit span \\
\hline CFT & Category Fluency Test \\
\hline COWAT & Controlled Word Association Test \\
\hline IL-6 & Interleukin-6 \\
\hline IL-2 & Interleukin-2 \\
\hline IL-4 & Interleukin-4 \\
\hline MIP2 & Microphage inflammatory protein-2 \\
\hline VCAM-1 & Vascular cell adhesion molecule-1 \\
\hline TNF-a & Tumor necrosis factor-alpha \\
\hline IL-1 $\beta$ & Interleukin 1-beta \\
\hline IFN-Y & Interferon- - -inducing factor \\
\hline HsCRP & High sensitive C-reactive protein \\
\hline CXCL & C-X-C chemokine ligand \\
\hline CCL & C-C chemokine ligand \\
\hline
\end{tabular}

\section{Declarations}

\section{Ethics approval and consent to participate}


This study was carried out in accordance with the 'Shanghai Mental Health Center ethical standards committee on human experimentation' with written informed consent from all subjects. All participants gave written informed consent in accordance with the Declaration of Helsinki. The protocol was approved by the 'Shanghai Mental Health Center ethical standards committee'. All subjects also gave written informed consent for the publication of this case report.

\section{Consent for publication}

Not applicable

\section{Competing interests}

The authors declare that the research was conducted in the absence of any commercial or financial relationships that could be construed as a potential conflict of interest.

\section{Funding}

This study was supported by grants of National Natural Science Foundation of China (No.81671402) and National Key R\&D Program of China(2017YFC1310500;2018YFC2002302).

\section{Author Contributions}

JN (1) performed statistical analysis and drafted the main manuscript text. YF (2), QQ (3), YL (4), LZ(5), $J Y Q(6)$ and $X L$ (7) performed the experiments and acquired the data. XL (8) were involved in study conception, participated in design and coordination, and helped to draft the manuscript. All the authors helped to draft the manuscript and gave critical comments. All the authors are acknowledged.

\section{Availability of data and materials}

All relevant raw data are freely available to any researchers who wish to use them for non-commercial purposes while preserving any necessary confidentiality and anonymity.

\section{Acknowledgements}

Not applicable

\section{References}


1. Livingston G, Sommerlad A, Orgeta V, Costafreda SG, Huntley J, Ames D, Ballard C, Banerjee S, Burns A, Cohen-Mansfield J et al: Dementia prevention, intervention, and care. The Lancet 2017, 390(10113):2673-2734.

2. Norton S, Matthews FE, Barnes DE, Yaffe K, Brayne C: Potential for primary prevention of Alzheimer's disease: an analysis of population-based data. Lancet Neurol 2014, 13(8):788-794.

3. Groeneweg-Koolhoven I, Ploeg M, Comijs HC, Wjh Penninx B, van der Mast RC, Schoevers RA, Rhebergen D, Exel EV: Apathy in early and late-life depression. J Affect Disord 2017, 223:76-81.

4. Linnemann C, Lang UE: Pathways Connecting Late-Life Depression and Dementia. Frontiers in Pharmacology 2020, 11.

5. Diniz BS, Butters MA, Albert SM, Dew MA, Reynolds CF: Late-life depression and risk of vascular dementia and Alzheimer's disease: systematic review and meta-analysis of community-based cohort studies. Br J Psychiatry 2013, 202(5):329-335.

6. Davis M, T OC, Johnson S, Cline S, Merikle E, Martenyi F, Simpson K: Estimating Alzheimer's Disease Progression Rates from Normal Cognition Through Mild Cognitive Impairment and Stages of Dementia. Curr Alzheimer Res 2018, 15(8):777-788.

7. Petersen RC, Stevens JC, Ganguli M, Tangalos EG, Cummings JL, DeKosky ST: Practice parameter: early detection of dementia: mild cognitive impairment (an evidence-based review). Report of the Quality Standards Subcommittee of the American Academy of Neurology. Neurology 2001, 56(9):1133-1142.

8. Jones KA, Thomsen C: The role of the innate immune system in psychiatric disorders. Mol Cell Neurosci 2013, 53:52-62.

9. Sperner-Unterweger B, Kohl C, Fuchs D: Immune changes and neurotransmitters: possible interactions in depression? Prog Neuropsychopharmacol Biol Psychiatry 2014, 48:268-276.

10. Lee KS, Chung JH, Lee KH, Shin MJ, Oh BH, Lee SH, Hong CH: Simultaneous measurement of 23 plasma cytokines in late-life depression. Neurol Sci 2009, 30(5):435-438.

11. Song F, Poljak A, Smythe GA, Sachdev P: Plasma biomarkers for mild cognitive impairment and Alzheimer's disease. Brain Res Rev 2009, 61(2):69-80.

12. Franciszkiewicz K, Boissonnas A, Boutet M, Combadiere C, Mami-Chouaib F: Role of chemokines and chemokine receptors in shaping the effector phase of the antitumor immune response. Cancer Res 2012, 72(24):6325-6332.

13. Milenkovic VM, Stanton EH, Nothdurfter C, Rupprecht R, Wetzel CH: The Role of Chemokines in the Pathophysiology of Major Depressive Disorder. Int J Mol Sci 2019, 20(9).

14. Koper OM, Kamińska J, Sawicki K, Kemona H: CXCL9, CXCL10, CXCL11, and their receptor (CXCR3) in neuroinflammation and neurodegeneration. Adv Clin Exp Med 2018, 27(6):849-856.

15. Hazen J VM, Barca ML, Eldholm RS, Persson K, Brækhus A, Saltvedt I, Selbæk G, Engedal K, Knapskog AB: The Association Between Circulating Inflammatory Markers and the Progression of Alzheimer Disease in Norwegian Memory Clinic Patients With Mild Cognitive Impairment or Dementia. Alzheimer Dis Assoc Disord 2020, 34(1):7. 
16. Kern S, Skoog I, Borjesson-Hanson A, Blennow K, Zetterberg H, Ostling S, Kern J, Gudmundsson P, Marlow T, Rosengren $L$ et al: Higher CSF interleukin- 6 and CSF interleukin-8 in current depression in older women. Results from a population-based sample. Brain Behav Immun 2014, 41:55-58.

17. Kim JM, Stewart R, Kim JW, Kang HJ, Bae KY, Kim SW, Shin IS, Yoon JS: Changes in proinflammatory cytokine levels and late-life depression: A two year population based longitudinal study. Psychoneuroendocrinology 2018, 90:85-91.

18. Marksteiner J, Kemmler G, Weiss EM, Knaus G, Ullrich C, Mechtcheriakov S, Oberbauer H, Auffinger S, Hinterholzl $\mathrm{J}$, Hinterhuber $\mathrm{H}$ et al: Five out of 16 plasma signaling proteins are enhanced in plasma of patients with mild cognitive impairment and Alzheimer's disease. Neurobiol Aging 2011, 32(3):539-540.

19. Petersen RC, Smith GE, Waring SC, Ivnik RJ, Tangalos EG, Kokmen E: Mild cognitive impairment: clinical characterization and outcome. Arch Neurol 1999, 56(3):303-308.

20. Tighe P, Negm $O$, Todd I, Fairclough L: Utility, reliability and reproducibility of immunoassay multiplex kits. Methods 2013, 61(1):23-29.

21. Zhang L, Hu XZ, Li X, Chen Z, Benedek DM, Fullerton CS, Wynn G, Biomarker t, Ursano RJ: Potential chemokine biomarkers associated with PTSD onset, risk and resilience as well as stress responses in US military service members. Trans/ Psychiatry 2020, 10(1):31.

22. Zlotnik A: Perspective: Insights on the Nomenclature of Cytokines and Chemokines. Front Immunol 2020, 11:908.

23. Stuart MJ, Baune BT: Chemokines and chemokine receptors in mood disorders, schizophrenia, and cognitive impairment: a systematic review of biomarker studies. Neurosci Biobehav Rev 2014, 42:93115.

24. Lai KSP, Liu CS, Rau A, Lanctot KL, Kohler CA, Pakosh M, Carvalho AF, Herrmann N: Peripheral inflammatory markers in Alzheimer's disease: a systematic review and meta-analysis of $\mathbf{1 7 5}$ studies. J Neurol Neurosurg Psychiatry 2017, 88(10):876-882.

25. Bradburn S, Sarginson J, Murgatroyd CA: Association of Peripheral Interleukin-6 with Global Cognitive Decline in Non-demented Adults: A Meta-Analysis of Prospective Studies. Front Aging Neurosci 2017, 9:438.

26. Ng A, Tam WW, Zhang MW, Ho CS, Husain SF, Mclntyre RS, Ho RC: IL-1beta, IL-6, TNF- alpha and CRP in Elderly Patients with Depression or Alzheimer's disease: Systematic Review and Meta-Analysis. Sci Rep 2018, 8(1):12050.

27. Leung R, Proitsi P, Simmons A, Lunnon K, Guntert A, Kronenberg D, Pritchard M, Tsolaki M, Mecocci P, Kloszewska I et al: Inflammatory proteins in plasma are associated with severity of Alzheimer's disease. PLoS One 2013, 8(6):e64971.

28. Jin JJ, Dai FX, Long ZW, Cai H, Liu XW, Zhou Y, Hong Q, Dong QZ, Wang YN, Huang H: CXCR6 predicts poor prognosis in gastric cancer and promotes tumor metastasis through epithelialmesenchymal transition. Oncol Rep 2017, 37(6):3279-3286. 
29. Stuart MJ, Singhal G, Baune BT: Systematic Review of the Neurobiological Relevance of Chemokines to Psychiatric Disorders. Front Cell Neurosci 2015, 9:357.

30. Sadik CD, Kim ND, Luster AD: Neutrophils cascading their way to inflammation. Trends Immunol 2011, 32(10):452-460.

31. Brosseron F, Krauthausen M, Kummer M, Heneka MT: Body fluid cytokine levels in mild cognitive impairment and Alzheimer's disease: a comparative overview. Mol Neurobio/ 2014, 50(2):534-544.

32. Shinko Y, Otsuka I, Okazaki S, Horai T, Boku S, Takahashi M, Ueno Y, Sora I, Hishimoto A: Chemokine alterations in the postmortem brains of suicide completers. J Psychiatr Res 2020, 120:29-33.

33. Conwell Y, Duberstein PR, Cox C, Herrmann J, Forbes N, Caine ED: Age differences in behaviors leading to completed suicide. Am J Geriatr Psychiatry 1998, 6(2):122-126.

34. Akturk $U$, Akturk S, Erci B: The effects of depression, personal characteristics, and some habits on physical activity in the elderly. Perspect Psychiatr Care 2019, 55(1):112-118.

35. Matthews MM, Hsu FC, Walkup MP, Barry LC, Patel KV, Blair SN: Depressive symptoms and physical performance in the lifestyle interventions and independence for elders pilot study. J Am Geriatr SoC 2011, 59(3):495-500.

36. Farioli Vecchioli S, Sacchetti S, Nicolis di Robilant V, Cutuli D: The Role of Physical Exercise and Omega-3 Fatty Acids in Depressive Illness in the Elderly. Curr Neuropharmacol 2018, 16(3):308-326.

37. Byeon H: Relationship between Physical Activity Level and Depression of Elderly People Living Alone. Int J Environ Res Public Health 2019, 16(20).

38. Beery AK, Zucker I: Sex bias in neuroscience and biomedical research. Neurosci Biobehav Rev 2011, 35(3):565-572.

39. Klein SL, Flanagan KL: Sex differences in immune responses. Nat Rev Immuno/2016, 16(10):626638.

40. Lucin $\mathrm{KM}$, Wyss-Coray $\mathrm{T}$ : Immune activation in brain aging and neurodegeneration: too much or too little?Neuron 2009, 64(1):110-122.

41. Zhang Y, Zheng Y, Xu Y, Sheng H, Ni X: Corticotropin-Releasing Hormone Suppresses Synapse Formation in the Hippocampus of Male Rats via Inhibition of CXCL5 Secretion by Glia. Endocrinology 2018, 159(2):622-638.

42. Forlani C MM, Ferrari B, Dalmonte E, Menchetti M, De Ronchi D, Atti AR. : Prevalence and gender differences in late-life depression: a population-based study. . Am J Geriatr Psychiatry 2014 22(4):10.

43. Tchalla AE WG, Sorond FA, Travison TG, Dantoine T, Lipsitz LA. : Elevated circulating vascular cell Adhesion Molecule-1 (sVCAM-1) is associated with concurrent depressive symptoms and cerebral white matter Hyperintensities in older adults. BMC Geriatr 2015, 4(15):7.

44. Nelson JC, Devanand DP: A systematic review and meta-analysis of placebo-controlled antidepressant studies in people with depression and dementia. J Am Geriatr Soc 2011, 59(4):577585 . 
45. Alexopoulos GS: Mechanisms and treatment of late-life depression. Trans/ Psychiatry 2019, 9(1):188.

Figures

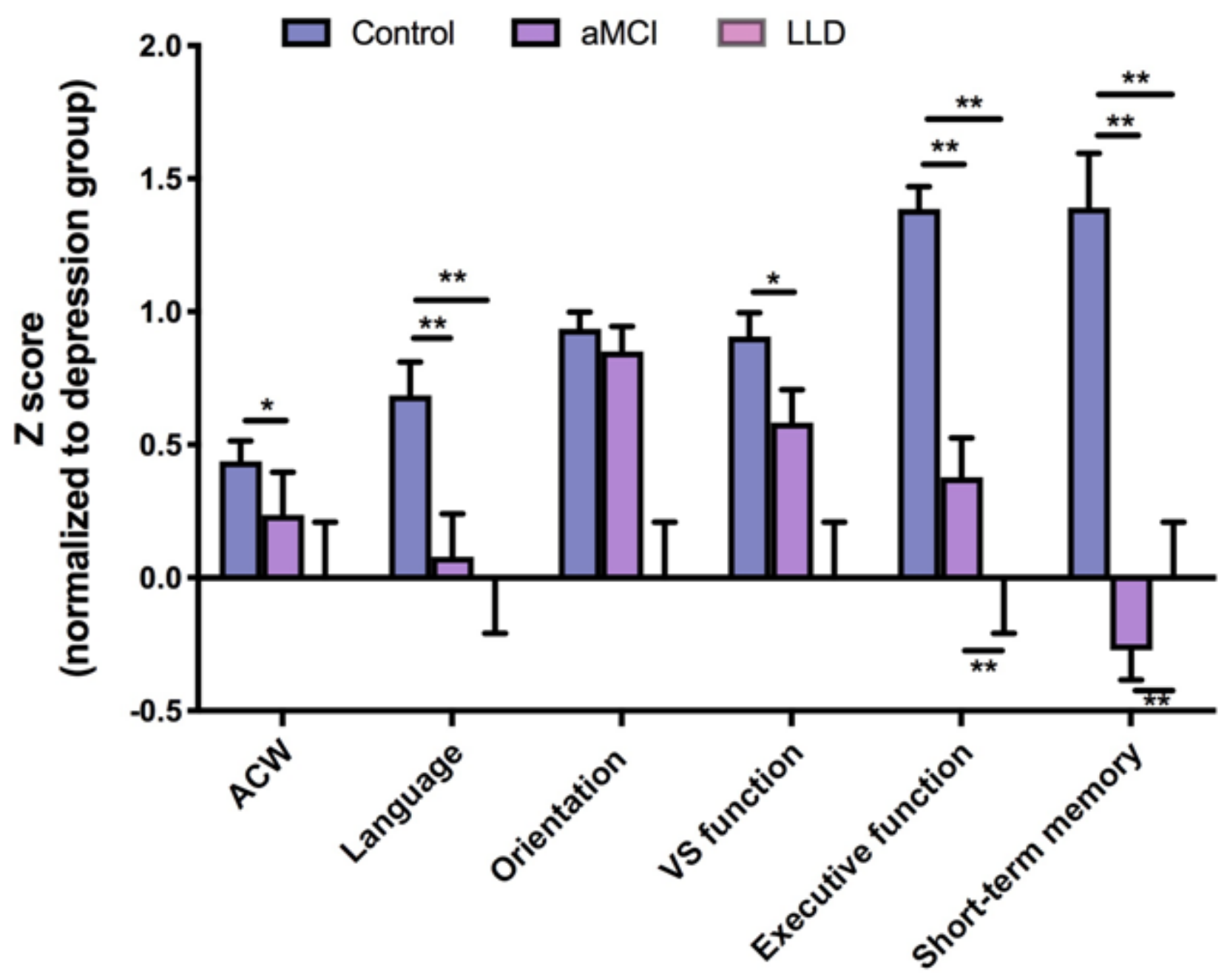

Figure 1

Z-Scores for the 6 different neurocognitive domains tested. Notes: aMCl, amnestic mild cognitive impairment; LLD, late life depression; Control, normal elderly; ACW, Attention, Concentration and Working memory; VS function, Visual spatial function;Significance levels are indicated on the upper portion of the figure. Error bars indicate standard error $\left({ }^{*} P<0.05,{ }^{*} P<0.01\right)$. 

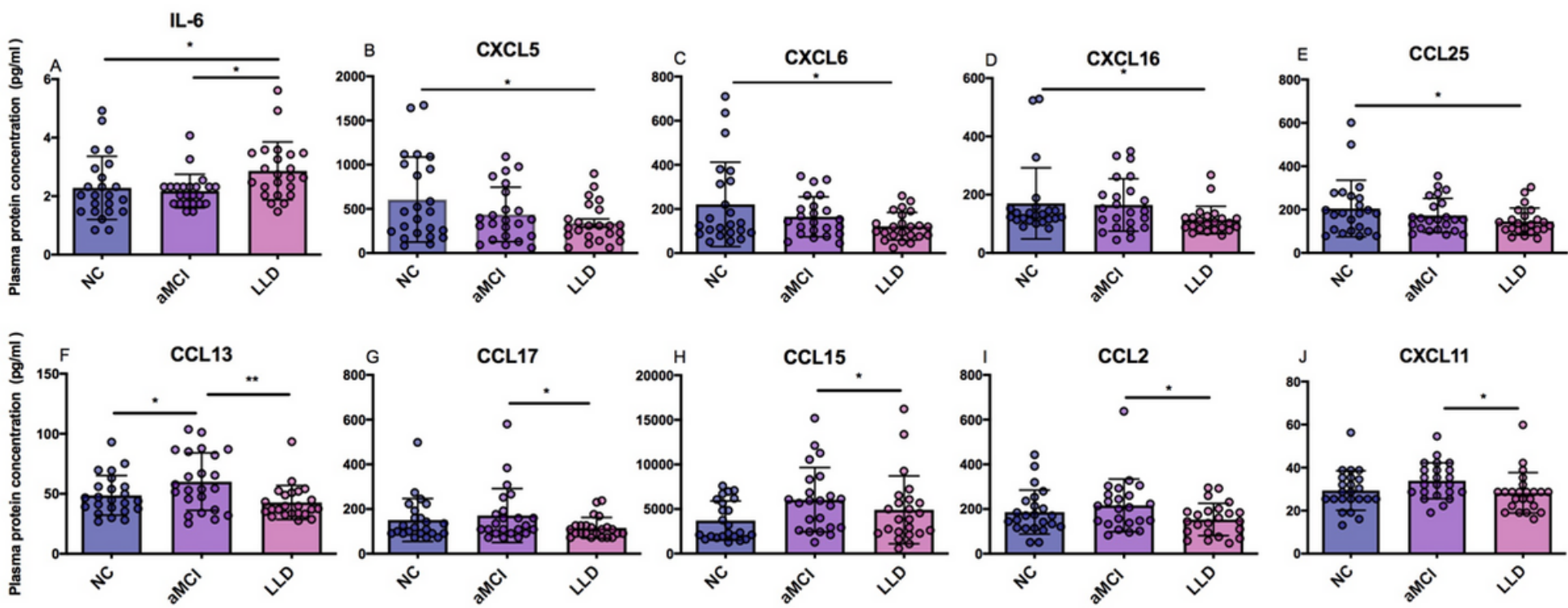

Figure 2

Chemokines and pro-inflammatory cytokines concentrations among patients with late life depression and amnestic mild cognitive impairment and normal controls with standard error of the mean. (A) CXCL5 among LLD, aMCl and control group. (B) CXCL6 among LLD, aMCl and control group. (C) CXCL11 among LLD, aMCl and control group. (D) CXCL16 among LLD, aMCl and control group. (E) CCL13 among LLD, aMCl and control group. (F) CCL15 among LLD, aMCl and control group. (G) CCL17 among LLD, aMCl and control group. (H) CCL25 among LLD, aMCl and control group. (I) CCL2 among LLD, aMCl and control group. (J) IL-6 among LLD, aMCl and control group. Notes: aMCl, amnestic mild cognitive impairment; LLD, late life depression; NC, normal control; IL-6, interleukin-6; CXCL5, C-X-C chemokine ligand 5;CXCL6, C-X-C chemokine ligand 6; CXCL11, C-X-C chemokine ligand 11; CXCL16, C-X-C chemokine ligand 16; CCL2, C-C chemokine ligand 2; CCL13, C-C chemokine ligand 13; CCL15, C-C chemokine ligand 15; CCL17, C-C chemokine ligand 17; CCL25, C-C chemokine ligand 25; Significance levels are indicated on the upper portion of the figure. $\left({ }^{\star} P<0.05,{ }^{\star *} P<0.01\right)$. 

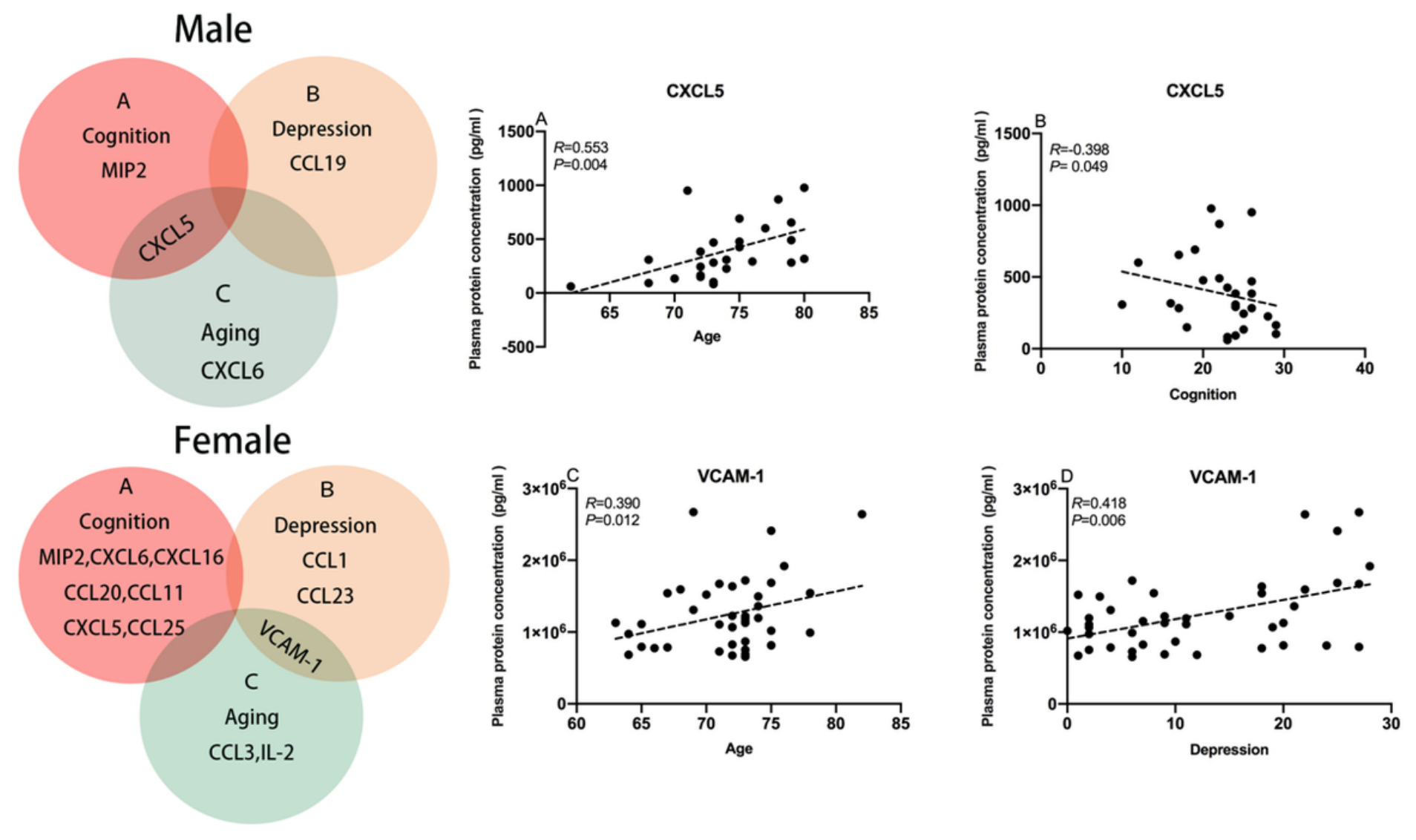

\section{Figure 3}

Venn diagram showing overlap of expression of potential chemokine markers for cognitive function, depression mood and aging with different gender (A-B) Association among CXCL5 protein level, C-MoCA total score and age in male. (C-D) Association amongVCAM-1 protein level GDS total score and age in female. Notes: MIP2,microphage inflammatory protein-2;CCL19, C-C chemokine ligand 19;CXCL6, C-X-C chemokine ligand 6; CXCL16, C-X-C chemokine ligand 16; CXCL5, C-X-C chemokine ligand 5; CCL1, C-C chemokine ligand $1 ;$ CCL11, C-C chemokine ligand 11; CCL20, C-C chemokine ligand 20; CCL23, C-C chemokine ligand 23; CCL25, C-C chemokine ligand 25; CCL3, C-C chemokine ligand 3;IL-2,interleukin 2; VCAM-1,vascular cell adhesion molecule-1; c-MoCA, Chinese Version ofMontrealCognitive Assessment; GDS,geriatric depression Scale.Both correlation coefficient (R) and P-values were presented.Only significant findings are shown. 
A

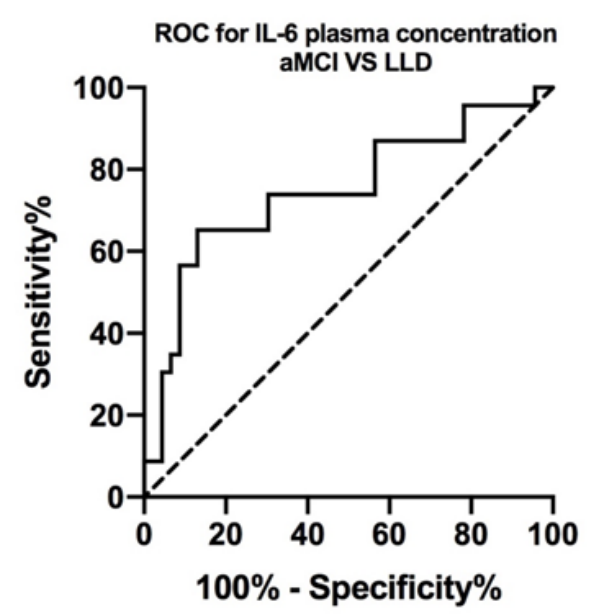

B

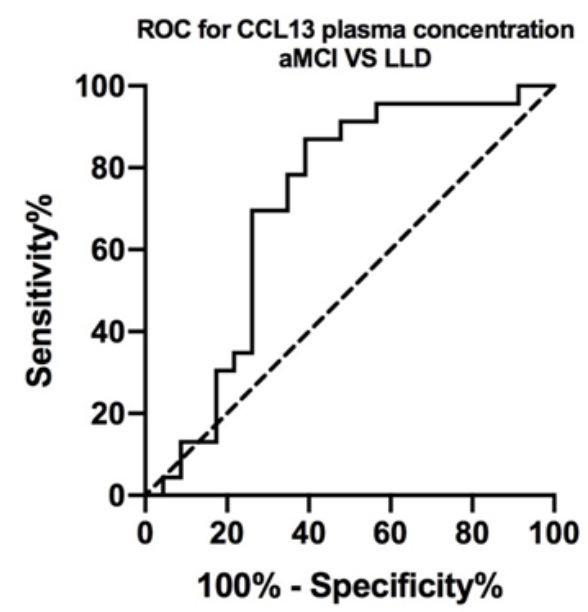

C

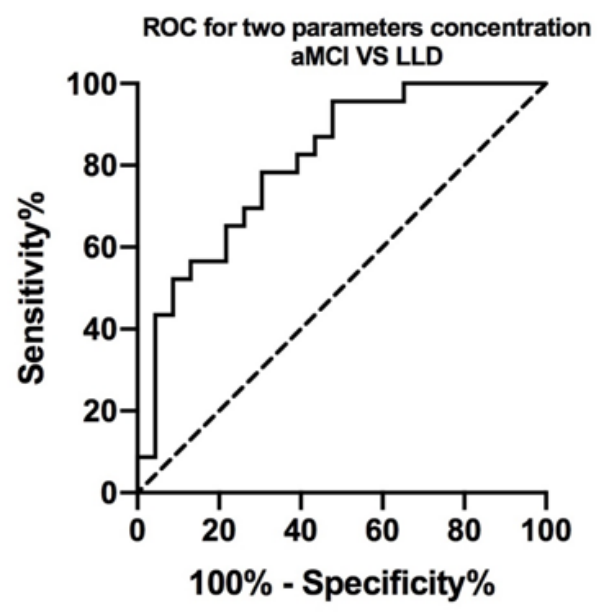

Figure 4

ROC curves for IL- 6 and CCL13 concentrations between patients with late life depression and amnestic mild cognitive impairment. (A) Receiver operating characteristic curve for IL-6 to discriminate between the aMCl and LLD groups (B) Receiver operating characteristic curve for CCL13 to discriminate between the aMCl and LLD groups (C) Receiver operating characteristic curve for combining IL-6and CCL13 to discriminate between the $\mathrm{aMCl}$ and LLD groups Notes: ROC = receiver operating characteristic. aMCl, amnestic mild cognitive impairment; LLD, late life depression; IL-6,interleukin-6; CCL13, C-C chemokine ligand 13 